\title{
EVALUACIÓN DE LA TOXICIDAD DE LOS SEDIMENTOS DEL CURSO ALTO DEL RÍO LERMA, MÉXICO
}

\author{
Carmen CARREÑO ${ }^{1}$, Graciela ZARAZÚA ${ }^{2 *}$, Cheikh FALL $^{3}$, \\ Pedro ÁVILA-PÉREZ ${ }^{2}$ y Samuel TEJEDA ${ }^{2}$
}

${ }^{1}$ División de Estudios de Posgrado e Investigación, Instituto Tecnológico de Toluca. Apartado Postal 890, Metepec, Estado de México, México, C. P. 52140

${ }^{2}$ Instituto Nacional de Investigaciones Nucleares. Carretera México-Toluca, Ocoyoacac, Estado de México, México, C. P. 52750

${ }^{3}$ Centro Interamericano de Recursos del Agua, Universidad Autónoma del Estado de México. Carretera TolucaAtlacomulco, kilómetro 14.5, Toluca, Estado de México, México, C. P. 50200

*Autor para correspondencia: graciela.zarazua@inin.gob.mx

(Recibido abril 2016; aceptado mayo 2017)

Palabras clave: microtox ${ }^{\circledR}$, contaminación en agua

\section{RESUMEN}

El objetivo de este estudio fue determinar la toxicidad de los sedimentos del curso alto del río Lerma (CARL), Estado de México, por medio del ensayo Microtox ${ }^{\circledR}$ con el protocolo básico en la fase sólida. Se colectaron muestras de sedimento superficial en 23 sitios distribuidos sobre el CARL: 12 sobre el cauce del río y 11 en sus tributarios, durante dos campañas de muestreo en un periodo de un año. La concentración efectiva media (EC50) en Vibrio fischeri varió para los sedimentos riverinos entre $139 \mathrm{mg} / \mathrm{L}$ y $3588 \mathrm{mg} / \mathrm{L}$ y para los de las descargas fue entre $108 \mathrm{mg} / \mathrm{L}$ y $16436 \mathrm{mg} / \mathrm{L}$. En general los sedimentos del CARL pueden clasificarse como extremadamente tóxicos, lo cual es un indicativo del impacto de las contribuciones antrópicas de origen doméstico e industrial que recibe este importante cuerpo de agua.

Key words: $\operatorname{microtox}{ }^{\circledR}$, water pollution

\begin{abstract}
The aim of this study was to evaluate the toxicity of sediments in the upper course of the Lerma River (UCLR), in the State of Mexico by means of Microtox ${ }^{\circledR}$ test using the basic solid phase test protocol. The surface sediment samples were collected in 23 sites distributed following the main stream flow of the river: 12 on the river and 11 on its tributaries for two sampling campaigns in a one year period. Half maximal effective concentration (EC50) in Vibrio fischeri varied in the river sediment from $139 \mathrm{mg} / \mathrm{L}$ to $3588 \mathrm{mg} / \mathrm{L}$ and for the tributaries it varied between $108 \mathrm{mg} / \mathrm{L}$ to $16436 \mathrm{mg} / \mathrm{L}$. In general, sediments of the UCLR can be classified as extremely toxics which show the impact of anthropogenic contributions that come from urban and industrial untreated discharges.
\end{abstract}




\section{INTRODUCCIÓN}

El sistema hidrológico Lerma-Chapala-Santiago es uno de los más importantes de México, con una superficie estimada de $130000 \mathrm{~km}^{2}$. Se divide en tres cuencas (alta, media y baja) y ocupa parte del Estado de México, Michoacán, Querétaro, Guanajuato y Jalisco. El curso alto del río Lerma (CARL) se ubica en la cuenca alta, en la región hidrológica 18, al este y noreste de la región hidrológica 26. Comprende la vertiente norte de la sierra Nahuatlaca-Matlazinca, así como la vertiente nororiental del Nevado de Toluca. Esta área queda comprendida desde las lagunas de Almoloya hasta $9 \mathrm{~km}$ aguas abajo de la presa José Antonio Alzate (GEM 2000 y 2009).

Según la clasificación de Köppen, el CARL presenta un clima $\mathrm{C}(\mathrm{w} 2)(\mathrm{w}) \mathrm{bi}(\mathrm{g})$, que corresponde a un clima semifrío subhúmedo, está formado por materiales volcánicos del terciario y cuaternario, las rocas aflorantes están representadas por materiales piroclásticos (tobas, aglomerados y brechas) y rocas de origen volcánico (basaltos y andesitas), las cuales por erosión y desintegración han formado arenas, gravas, limos y depósitos lacustres. Los suelos que predominan son tipo feozem gleyco de textura media, vertisoles pélicos de textura fina resultado de la antigua zona lacustre y litosoles con textura media en una fracción de la Sierra Nahuatlaca-Matlazinca (GEM 1993 y 1997).

El valle de Toluca, donde se localiza el CARL, es una zona industrialmente activa con una producción agrícola importante y un acelerado crecimiento urbano e industrial. En esta zona hasta el año 2000 se contaba con 108 plantas de tratamiento para aguas residuales de origen industrial, algunas de las cuales concluyen su tratamiento en las macroplantas como Reciclagua, Toluca-Norte y Toluca-Oriente, con un gasto total de operación de 2470 L/s (GEM 2000, 2002 y 2009).

La zona metropolitana del valle de Toluca (ZMVT) descarga sus aguas residuales en colectores ( $7 \%$ ), campos, pozos de absorción y subsuelo ( $27 \%$ ), canales $(31 \%)$ y ríos y arrollos (35\%), de las cuales más de la mitad tributan al río Lerma. Así, este cuerpo de agua se ha convertido en el principal colector de los desechos industriales y domésticos con un alto contenido de materia orgánica, compuestos nitrogenados, metales pesados y otras sustancias químicas tóxicas, que no se degradan fácilmente en condiciones naturales (Tejeda et al. 2006, Zarazúa 2008, Ávila et al. 2011, Zarazúa et al. 2011).

En un ecosistema acuático se puede considerar que existen tres reservorios para los contaminantes que son el agua, el sedimento y la biota, en este sentido los sedimentos son importantes ya que las concentraciones más altas se encuentran generalmente en el agua intersticial o asociadas con la fase sólida de éstos. Por ello, el análisis de los sedimentos juega un papel importante en estudios ambientales ya que son receptores de contaminantes, los cuales no permanecen fijos sino que pueden ser removidos por agentes biológicos y químicos (Hansen et al. 1995, Tarvainen et al. 1997, Pedroza et al. 2010). En general, las sustancias potencialmente tóxicas presentes en el sedimento no producen un efecto tóxico agudo en los organismos bentónicos. La mayoría de los individuos presentan respuestas subletales (deformidades morfológicas, daño en el crecimiento o reproducción), también puede observarse con la presencia de sustancias tóxicas: la movilización de organismos, la bioacumulación y la magnificación (Bryan y Hummerstone 1978, Bryan y Uysal 1978, Luoma y Bryan 1979, Walton et al. 1982, Butterworth et al. 1995).

El estudio de los cuerpos de agua se vuelve más complejo cuando están contaminados, debido a que al interactuar las sustancias antrópicas con las geogénicas, cambian las propiedades físicas y químicas del ecosistema acuático (oxígeno disuelto, $\mathrm{pH}$, temperatura, demanda biológica y química de oxígeno, etc.) que alteran la calidad del agua y pueden representar un riesgo toxicológico. Por ello, es importante conocer cómo la toxicidad de los sedimentos contribuye con la degradación de los cuerpos de agua a través de la valoración de bioensayos de toxicidad (Niffeler et al. 1984).

La medida de toxicidad de las aguas residuales y cuerpos de agua, es una parte importante del monitoreo de la contaminación ambiental ya que permite la incorporación de este parámetro a un marco regulatorio. Durante los últimos años, el bioensayo de toxicidad, distribuido comercialmente como Microtox ${ }^{\circledR}$ se ha utilizado ampliamente para evaluar la toxicidad de muestras ambientales debido a sus bondades en cuanto a sensibilidad, poder discriminante, reproducibilidad y fácil aplicación. El ensayo Microtox ${ }^{\circledR}$ determina la toxicidad aguda en aguas naturales y residuales, así como en muestras sólidas (sedimentos, suelos y sus extractos acuosos), la cual se expresa como la concentración $(\%$ o $\mathrm{mg} / \mathrm{kg})$ de agentes contaminantes que produce la reducción de $50 \%$ de la luminiscencia inicial también conocida como concentración efectiva media (EC50) de la bacteria marina Vibrio fischeri. El resultado indica que a una mayor concentración hay una menor mortandad de esta bacteria y por lo tanto menor toxicidad de 
los contaminantes que están presentes en la muestra analizada (Azur 1992, Jennings et al. 2001, Hsieha et al. 2004, Tsiridis et al. 2006). El bioensayo Microtox ${ }^{\circledR}$ ha mostrado mayor sensibilidad y una buena correlación con los resultados obtenidos en peces y en Daphnia magna, siendo desde 1984 una de las pruebas recomendadas por la Agencia de Protección Ambiental (EPA, por sus siglas en inglés) para la evaluación de la toxicidad aguda de muestras de agua (Bennett y Cubbage 1992, Azur 1998, Beelen 2003, Onorati y Mecozzi 2004, Burga-Pérez et al. 2012).

Actualmente en la ZMVT el gran número de industrias asentadas y el enorme crecimiento de la zona urbana han provocado la sobreexplotación de los mantos acuíferos y la contaminación de los cuerpos de agua por aguas residuales y escurrimientos agrícolas (GEM 2000 y 2002). El objetivo del presente trabajo fue evaluar la toxicidad de los sedimentos del curso alto del río Lerma y sus principales tributarios mediante el ensayo Microtox ${ }^{\circledR}$ en dos épocas del año, con el fin de servir de base para la toma de decisiones en el control de la contaminación de este importante sistema hidrológico.

\section{MATERIALES Y MÉTODOS}

\section{Área de estudio}

El curso alto del río Lerma (CARL) donde se llevó a cabo el presente estudio, se ubica geográficamente entre los paralelos $19^{\circ} 03^{\prime} 40^{\prime \prime}$ y $19^{\circ} 30^{\prime} 21^{\prime \prime}$ de latitud norte y los meridianos 99 $26^{\circ} 20^{\prime \prime}$ y $99^{\circ} 38^{\prime} 47^{\prime \prime}$ de longitud oeste (Fig. 1; SMA 2006). Tiene una superficie de $2117.88 \mathrm{~km}^{2}$, una longitud de $50.2 \mathrm{~km}$, una altitud media de $2848 \mathrm{msnm}$ y una pendiente media de $8.54 \%$. La temporada lluviosa comienza a mediados de mayo y termina a mediados de octubre, con lluvias de verano y un porcentaje de lluvia invernal menor a $5 \%$, la precipitación media anual supera los 800 mm de lluvia (Díaz et al. 2005).

\section{Muestreo}

Se seleccionaron 23 sitios de muestreo: 12 sobre el río (R) y 11 sobre tributarios $(\mathrm{T})$ que descargan al CARL (Fig. 1). Para definir los sitios de muestreo se tomó como base las estaciones de monitoreo establecidas por la Comisión Nacional del Agua y el Centro Interamericano de Recursos del Agua, las cuales se ubican en las principales zonas de afectación a este cuerpo de agua (Fall et al. 2007).

Se realizaron dos muestreos durante un ciclo hidrológico (estiaje y lluvias). Las muestras se tomaron de acuerdo con lo propuesto en la norma mexicana NMX-AA-112-1995-SCFI (SCOFI 1995). El sedimento se tomó del centro de cada sitio con una draga tipo ponar de acero inoxidable y se colocaron en frascos de polietileno de alta densidad con el cuidado de llenarlos totalmente para evitar espacios entre la muestra y la tapa. Debido a que la toxicidad de las muestras puede cambiar con el tiempo se almacenaron a $4 \pm 2{ }^{\circ} \mathrm{C}$ para su traslado al laboratorio y se analizaron dentro de las primeras 48 horas.

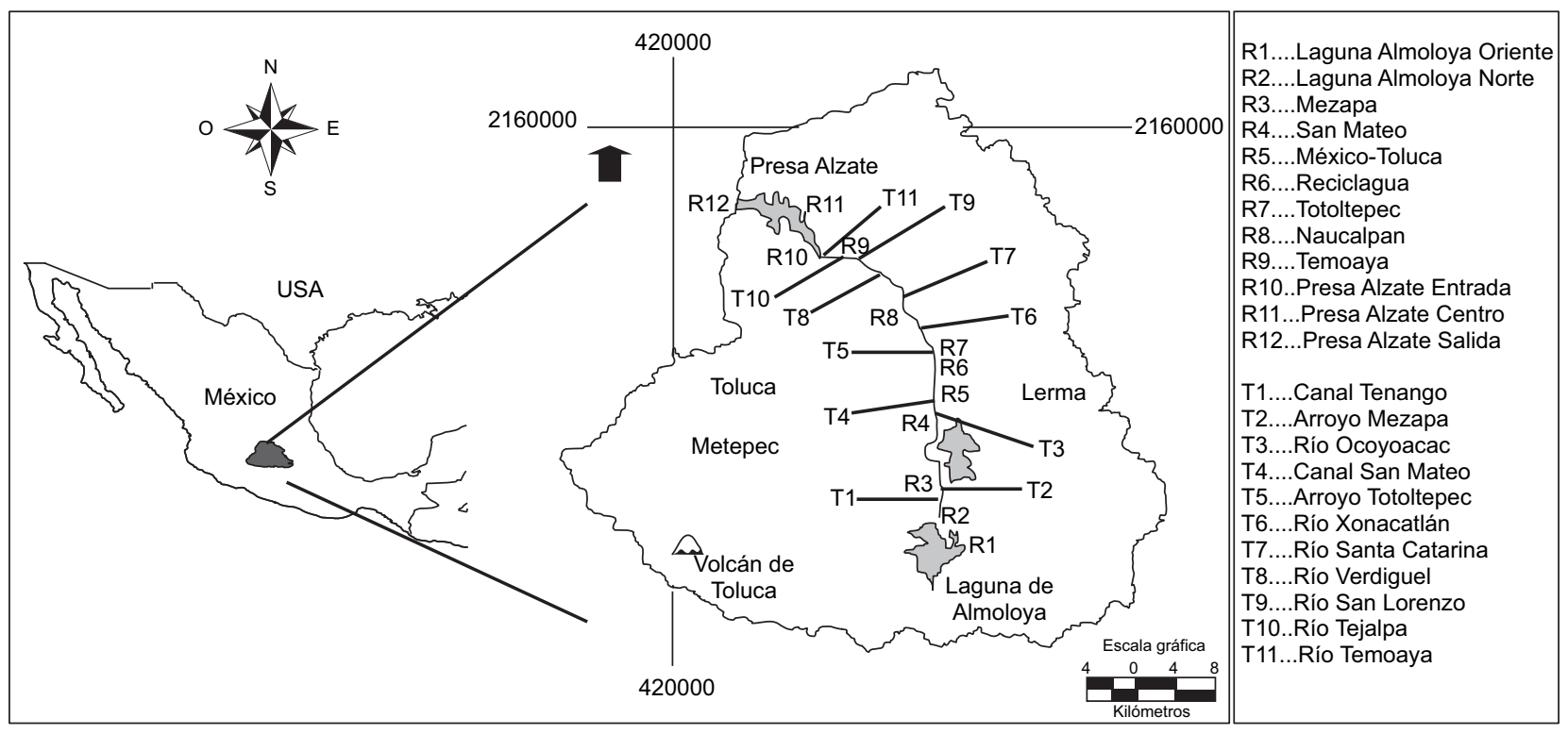

Fig. 1. Localización del curso alto del río Lerma, México y sitios de muestreo de sedimentos sobre el río (R) y en los tributarios (T) 


\section{Análisis físicos y químicos de los sedimentos}

A cada muestra de sedimento se le determinó el pH con un potenciómetro marca "Hach" (SCOFI 1984) y el contenido de carbono orgánico total (COT) con un analizador elemental "OI Analytical". Se seleccionaron estos parámetros ya que pueden influir en la toxicidad y movilidad de los contaminantes ( $\mathrm{Coz}$ et al. 2008, Karlsson et al. 2010).

\section{Análisis de la toxicidad de los sedimentos por Microtox ${ }^{\circledR}$}

Los sedimentos pueden ser analizados con el ensayo en la fase sólida (SPT, por sus siglas en inglés), el cual fue desarrollado por AZUR ${ }^{\circledR}$ (1998) y ha sido utilizado durante los últimos años en distintos estudios para evaluar toxicidad aguda en sedimentos (Riba et al. 2004, Campisi et al. 2005, MoralesCaselles et al. 2006, Mamindy-Pajany et al. 2011 y 2012, Maranho et al. 2015a, 2015b). El protocolo SPT mide la emisión de la luz de las bacterias después de su incubación en contacto directo con una mezcla de sedimento y $\mathrm{NaCl}$ al $2 \%$.

En este estudio se aplicó el protocolo básico en la fase sólida (BSPT, por sus siglas en inglés), el cual es más simple ya que suprime la filtración e incorpora el contacto directo de las bacterias con las partículas de sedimento, así como con el agua intersticial al medir la emisión de luz cuando la bacteria está aún en contacto con el sedimento, por lo que esta emisión de luz no se ve afectada por las bajas tasas de recuperación de las bacterias (Azur 1998, CasadoMartínez et al. 2006).

Las muestras se analizaron en un equipo Microtox ${ }^{\circledR}$ marca SDI, modelo M500 bajo las siguientes condiciones: A $5 \mathrm{~g}$ de sedimento homogeneizado se le adicionaron $20 \mu \mathrm{L}$ de la cepa Vibrio fischeri (conocida anteriormente como Photobacterium phosphoreum, NRRL B-11177) reconstituida con una solución no tóxica de $\mathrm{NaCl}$ al $2 \%$ y se dejó incubar 20 minutos a $5.5 \pm 1{ }^{\circ} \mathrm{C}$. Posteriormente se transfirieron $500 \mu \mathrm{L}$ a una celdilla de análisis y se leyó a $490 \pm 100 \mathrm{~nm}$ a una temperatura ambiente de $15 \pm 0.5^{\circ} \mathrm{C}$. A través del programa MTX-Microtox ${ }^{\circledR}$ se obtuvieron los cálculos para determinar la EC50 expresada como la concentración (\%) de elementos y/o compuestos que pueden ser inhibitorios de la bioluminiscencia para V. fischeri. El intervalo de confianza considerado fue de $95 \%$ (Azur 1992).

\section{Análisis estadístico}

Los datos se procesaron mediante el programa Statgraphics plus V. 5 para obtener los valores promedio, desviación estándar y límites de confianza.
Además, se realizó una prueba de normalidad de Shapiro-Wilks. Se aplicó un análisis de varianza para los resultados que presentaron normalidad y uno de Kruskal-Wallis para los no normales entre los sitios y épocas de muestreo. Los diferentes análisis estadísticos se consideraron con un intervalo de confianza de $95 \%(\alpha \leq 0.05)$.

\section{RESULTADOS Y DISCUSIÓN}

Los cuadros I y II muestran los valores de $\mathrm{pH}$ y la concentración del COT en los sedimentos correspondientes al lecho del río y sobre los tributarios muestreados. En el caso del pH, sólo los sedimentos de la laguna de Almoloya fueron ligeramente básicos,

CUADRO I. pH Y CARBONO ORGÁNICO TOTAL DE LOS SEDIMENTOS SOBRE EL RÍO (R) DEL CURSO ALTO DEL RÍO LERMA

\begin{tabular}{llcc}
\hline Sitio de muestreo & & $\mathrm{pH}$ & $\begin{array}{c}\text { Carbono } \\
\text { orgánico total } \\
\text { (\% en peso) }\end{array}$ \\
\hline Laguna Almoloya Oriente & $\mathrm{R} 1$ & 8.15 & 15.1 \\
Laguna Almoloya Norte & $\mathrm{R} 2$ & 8.21 & 15.2 \\
Mezapa & $\mathrm{R} 3$ & 7.28 & 11.1 \\
San Mateo & $\mathrm{R} 4$ & 7.22 & 7.4 \\
México-Toluca & $\mathrm{R} 5$ & 7.20 & 5.4 \\
Reciclagua & $\mathrm{R} 6$ & 7.18 & 7.5 \\
Totoltepec & $\mathrm{R} 7$ & 7.41 & 7.2 \\
Naucalpan & $\mathrm{R} 8$ & 7.43 & 7.5 \\
Temoaya & $\mathrm{R} 9$ & 7.28 & 3.5 \\
Presa Alzate entrada & $\mathrm{R} 10$ & 7.31 & 6.2 \\
Presa Alzate centro & R11 & 7.18 & 3.9 \\
Presa Alzate salida & R12 & 7.11 & 0.8 \\
\hline
\end{tabular}

CUADRO II. pH Y CARBONO ORGÁNICO TOTAL DE LOS SEDIMENTOS DE LOS TRIBUTARIOS (T) AL CURSO ALTO DEL RÍO LERMA

\begin{tabular}{llcc}
\hline Sitio de muestreo & & $\mathrm{pH}$ & $\begin{array}{c}\text { Carbono orgánico } \\
\text { total }(\% \text { en peso) }\end{array}$ \\
\hline Canal Tenango & $\mathrm{T} 1$ & 7.22 & 7.1 \\
Arroyo Mezapa & $\mathrm{T} 2$ & 7.28 & 10.6 \\
Río Ocoyoacac & $\mathrm{T} 3$ & 7.19 & 6.8 \\
Canal San Mateo & $\mathrm{T} 4$ & 7.24 & 7.2 \\
Arroyo Totoltepec & $\mathrm{T} 5$ & 7.23 & 7.6 \\
Río Xonacatlán & $\mathrm{T} 6$ & 7.19 & 3.1 \\
Río Santa Catarina & $\mathrm{T} 7$ & 7.17 & 2.3 \\
Río San Lorenzo & $\mathrm{T} 8$ & 7.21 & 3.2 \\
Río Verdiguel & $\mathrm{T} 9$ & 7.43 & 6.4 \\
Río Tejalpa & $\mathrm{T} 10$ & 7.22 & 3.2 \\
Río Temoaya & $\mathrm{T} 11$ & 7.27 & 2.6 \\
\hline
\end{tabular}


entre 8.15 y 8.21 , mientras que para los demás sedimentos este parámetro varió entre 7.11 y 7.43. La concentración de COT, en el sedimento del lecho del río varió entre $0.8 \%$ y $16.2 \%$ y en los tributarios entre $2.65 \%$ y $10.6 \%$ (Cuadros I y II). Los sedimentos con mayor concentración promedio fueron Almoloya (R1 y R2) y Mezapa (R3 y D2), en tanto que los de menor concentración promedio fueron los de la presa Alzate a su salida y los correspondientes a los ríos Xonacatlán, Santa Catarina, San Lorenzo, Tejalpa y Temoaya.

En los cuadros III y IV se muestran los resultados promedio de EC50 de las 23 muestras correspondientes a los sedimentos de río y tributarios al CARL. Para los primeros, la concentración de la EC50 varió entre $139 \mathrm{mg} / \mathrm{kg}$ y $3588 \mathrm{mg} / \mathrm{kg}$ y para las descargas fue

CUADRO III. CONCENTRACIÓN EFECTIVA MEDIA (EC50) DE LOS SEDIMENTOS SOBRE EL RÍO (R) DEL CURSO ALTO DEL RÍO LERMA OBTENIDA POR EL MÉTODO DE MICROTOX®

\begin{tabular}{|c|c|c|c|c|c|c|}
\hline \multirow{3}{*}{ Sitio de muestreo } & & \multicolumn{5}{|c|}{ Temporada } \\
\hline & & \multicolumn{2}{|c|}{ Estiaje } & \multicolumn{2}{|c|}{ Lluvia } & \multirow{2}{*}{$\begin{array}{c}\text { Media } \\
\begin{array}{c}\mathrm{EC}_{50} \\
(\mathrm{mg} / \mathrm{kg})\end{array}\end{array}$} \\
\hline & & $\begin{array}{c}\mathrm{EC}_{50} \\
(\mathrm{mg} / \mathrm{kg})\end{array}$ & $\begin{array}{c}\mathrm{EC}_{50} \\
(\%)\end{array}$ & $\begin{array}{c}\mathrm{EC}_{50} \\
(\mathrm{mg} / \mathrm{kg})\end{array}$ & $\begin{array}{c}\mathrm{EC}_{50} \\
(\%)\end{array}$ & \\
\hline Laguna Almoloya Oriente & $\mathrm{R} 1$ & $969 \pm 95$ & 0.0969 & $1087 \pm 83$ & 0.1017 & 993 \\
\hline Laguna Almoloya Norte & $\mathrm{R} 2$ & $409 \pm 38$ & 0.0409 & $715 \pm 55$ & 0.0715 & 562 \\
\hline Mezapa & R3 & $180 \pm 19$ & 0.0180 & $164 \pm 16$ & 0.0164 & 172 \\
\hline San Mateo & $\mathrm{R} 4$ & $234 \pm 21$ & 0.0234 & $279 \pm 25$ & 0.0279 & 257 \\
\hline México-Toluca & R5 & $225 \pm 19$ & 0.0225 & $145 \pm 13$ & 0.0145 & 185 \\
\hline Reciclagua & R6 & $139 \pm 12$ & 0.0139 & $152 \pm 12$ & 0.0152 & 146 \\
\hline Totoltepec & R7 & $147 \pm 13$ & 0.0147 & $223 \pm 19$ & 0.0223 & 185 \\
\hline Naucalpan & $\mathrm{R} 8$ & $223 \pm 18$ & 0.0223 & $501 \pm 32$ & 0.0501 & 362 \\
\hline Temoaya & R9 & $2675 \pm 115$ & 0.2675 & $3588 \pm 97$ & 0.3588 & 3132 \\
\hline Presa Alzate entrada & $\mathrm{R} 10$ & $350 \pm 28$ & 0.0350 & $213 \pm 18$ & 0.0213 & 282 \\
\hline Presa Alzate centro & $\mathrm{R} 11$ & $283 \pm 25$ & 0.0283 & $225 \pm 20$ & 0.0225 & 254 \\
\hline Presa Alzate salida & $\mathrm{R} 12$ & $964 \pm 87$ & 0.0964 & $1040 \pm 79$ & 0.1040 & 1002 \\
\hline Máximo & & 2675 & 0.2675 & 3588 & 0.3588 & \\
\hline Mínimo & & 139 & 0.0139 & 145 & 0.0145 & \\
\hline
\end{tabular}

CUADRO IV. CONCENTRACIÓN EFECTIVA MEDIA (EC50) DE LOS SEDIMENTOS DE LOS TRIBUTARIOS (T) AL CURSO ALTO DEL RÍO LERMA OBTENIDA POR EL MÉTODO DE MICROTOX®

\begin{tabular}{|c|c|c|c|c|c|c|}
\hline \multirow{3}{*}{ Sitio de Muestreo } & & \multicolumn{5}{|c|}{ Temporada } \\
\hline & & \multicolumn{2}{|c|}{ Estiaje } & \multicolumn{2}{|c|}{ Lluvia } & \multirow{2}{*}{$\frac{\text { Media }}{\begin{array}{c}\mathrm{EC}_{50} \\
(\mathrm{mg} / \mathrm{kg})\end{array}}$} \\
\hline & & $\begin{array}{c}\mathrm{EC}_{50} \\
(\mathrm{mg} / \mathrm{kg})\end{array}$ & $\begin{array}{c}\mathrm{EC}_{50} \\
(\%)\end{array}$ & $\begin{array}{c}\mathrm{EC}_{50} \\
(\mathrm{mg} / \mathrm{kg})\end{array}$ & $\begin{array}{c}\mathrm{EC}_{50} \\
(\%)\end{array}$ & \\
\hline Canal Tenango & $\mathrm{T} 1$ & $243 \pm 21$ & 0.0243 & $260 \pm 22$ & 0.0260 & 252 \\
\hline Arroyo Mezapa & $\mathrm{T} 2$ & $108 \pm 11$ & 0.0108 & $211 \pm 20$ & 0.0211 & 160 \\
\hline Río Ocoyoacac & $\mathrm{T} 3$ & $629 \pm 54$ & 0.0629 & $845 \pm 77$ & 0.0845 & 737 \\
\hline Canal San Mateo & $\mathrm{T} 4$ & $334 \pm 28$ & 0.0334 & $329 \pm 27$ & 0.0329 & 332 \\
\hline Arroyo Totoltepec & T5 & $943 \pm 43$ & 0.0943 & $822 \pm 63$ & 0.0822 & 883 \\
\hline Río Xonacatlán & $\mathrm{T} 6$ & $3279 \pm 102$ & 0.3279 & $3770 \pm 144$ & 0.3770 & 3525 \\
\hline Río Santa Catarina & $\mathrm{T} 7$ & $8896 \pm 157$ & 0.8896 & $7296 \pm 176$ & 0.7296 & 8096 \\
\hline Río San Lorenzo & $\mathrm{T} 8$ & $10150 \pm 184$ & 1.0150 & $16436 \pm 203$ & 1.6436 & 13293 \\
\hline Río Verdiguel & T9 & $1708 \pm 95$ & 0.1708 & $2122 \pm 113$ & 0.2122 & 1915 \\
\hline Río Tejalpa & $\mathrm{T} 10$ & $5623 \pm 115$ & 0.5623 & $6397 \pm 125$ & 0.6397 & 6010 \\
\hline Río Temoaya & $\mathrm{T} 11$ & $13090 \pm 187$ & 1.3090 & $12961 \pm 201$ & 1.2961 & 13026 \\
\hline Máximo & & 13090 & 1.309 & 16436 & 1.6436 & \\
\hline Mínimo & & 108 & 0.0108 & 188 & 0.0188 & \\
\hline
\end{tabular}


entre $108 \mathrm{mg} / \mathrm{kg}$ y $16436 \mathrm{mg} / \mathrm{kg}$. Existen diferentes criterios para evaluar la toxicidad de los sedimentos debido a la presencia de contaminantes, como metales $(\mathrm{Ni}, \mathrm{Cu}, \mathrm{Zn}, \mathrm{As}, \mathrm{Cd}, \mathrm{Pb}$ ) y compuestos orgánicos persistentes (hidrocarburos aromáticos policíclicos y los bifenilos policlorados) que pueden ser inhibitorios del metabolismo de V. fischeri. Kwan y Dutka (1995 y 1996) estandarizaron la prueba de Microtox ${ }^{\circledR}$ para sedimentos considerando los resultados expesados como EC50 en \% y los clasificaron en tres grupos: 1) extremadamente tóxicos $(\mathrm{EC} 50<0.5 \%), 2)$ moderadamente tóxicos $(0.5<$ EC50 $\leq 1 \%)$ y 3$)$ no tóxicos $(\mathrm{EC} 50>1.0 \%)$. De acuerdo con este criterio todos los sedimentos sobre CARL pueden ser considerados como extremadamente tóxicos al igual que los sedimentos de los tributarios a excepción de los correspondientes a los ríos Santa Catarina, Tejalpa (moderadamente tóxicos), Temoaya y San Lorenzo (no tóxicos). De acuerdo con el criterio de Doe et al. (2005), para valores expresados en $\mathrm{mg} / \mathrm{kg}$, si el valor de EC50 es $\leq 1000 \mathrm{mg} / \mathrm{kg}$, entonces los sedimentos se consideran tóxicos, mientras que si el valor es $>1000 \mathrm{mg} / \mathrm{kg}$, entonces se consideran no tóxicos. Con base en lo anterior, sólo el sedimento riverino de Temoaya y Presa Alzate (salida) y los de los ríos Xonacatlán, Santa Catarina, San Lorenzo, Verdiguel, Tejalpa y Temoaya se pueden considerar como no tóxicos.

Los resultados del análisis de varianza de la $\mathrm{EC}_{50}$ en los sedimentos riverinos, muestran que en general las muestras tomadas de los sitios de Temoaya, Presa Alzate (salida) y Almoloya (norte y oriente) son estadísticamente diferentes al resto de los sedimentos $(\alpha \leq$ 0.05 ), lo cual puede deberse a que los sitios en la parte intermedia del CARL tienen una fuerte influencia de descargas tanto de origen municipal como industrial. Por otro lado, la laguna de Almoloya recibe un aporte importante de aguas municipales no tratadas de las poblaciones aledañas, presenta una pendiente de sur a norte y una barrera física que impide que el agua fluya hacia lo que es el inicio del río Lerma, por lo que probablemente muchos de los contaminantes se están depositando en esta zona y así, provocan la alteración de la calidad de los sedimentos (Tejeda et al. 2005, 2006, Zarazúa et al. 2011). El resultado de este estudio clasifica a los sedimentos de esta laguna como extremadamente tóxicos, con un $\mathrm{pH}$ ligeramente básico, lo cual se puede deber a la naturaleza calcoalcalina de la zona, constituida principalmente por materiales piroclásticos y rocas basálticas (GEM 1993, Díaz et al. 2005). La concentración promedio de COT fue de $15 \%$, con lo que se rebasó el límite máximo recomendado (10\%) por el Ministerio de
Ambiente y Energía de Ontario para la protección de la vida acuática (MEEO 1993). Además, se presentan altas concentraciones de compuestos orgánicos, metales, materia orgánica, nutrientes y un abatimiento de los niveles de oxígeno disuelto (Hinojosa 2006, Carreño 2008, Zarazúa 2008).

Las condiciones físicas y químicas antes mencionadas, pueden ocasionar que la biota que aún habita en esta laguna presente efectos tóxicos de ligeros a severos, como la alteración en la reproducción y en el metabolismo. Lo cual se puede ver reflejado principalmente en la disminución del número de huevos incubados y tiempo de vida, retraso en la maduración sexual y crecimiento, daño enzimático y citológico, hipoxia e incremento de la frecuencia respiratoria (Kubitz et al. 1995, Suedel et al. 1996, Bernet et al. 2004, Hattink et al. 2006, Levesque et al. 2006, Talapatra y Banerjee 2007). A partir del sitio de Mezapa hasta Naucalpan, los sedimentos se clasificaron como extremadamente tóxicos lo cual se puede deber a que esta zona del río está altamente impactada por contribuciones antrópicas, principalmente industriales de diverso origen como metal-mecánico, alimentario, textil, pintura, calzado, plantas de tratamiento, etc. (Zarazúa 2008, Ávila 2011). El pH promedio de estos sedimentos fue de 7.30 y la concentración más alta de COT se presentó en el sitio de Mezapa (10.6 $\%)$, lo anterior se debe a que en esta zona ingresan al río desechos orgánicos de origen animal provenientes principalmente de rastros de ganado ovino, bovino, porcino y aves de corral (Zarazúa 2008). Estudios previos reportan en el agua y sedimento de esta zona del CARL, compuestos orgánicos persistentes (COP) entre los cuales se encuentran el nitrobenceno, terfenil, 2 fluorobifenil, fenol d6, 2 fluorofenol, 2,4,6 tribromofenol, entre otros, así como los metales cromo, cobre, zinc y plomo, todos ellos considerados como tóxicos para la biota acuática (Tejeda et al. 2005 y 2006, Zarazúa et al. 2006, Carreño 2008, Zarazúa 2008). Debido a las condiciones fisicas y químicas de este cuerpo de agua, la anoxia, la alta concentración de la demanda química y bioquímica de oxígeno (Hinojosa 2006, Zarazúa 2008), es que los contaminantes orgánicos persistentes y los metales pueden asociarse a otros compuestos y/o elementos, formando compuestos insolubles y así, depositarse en el lecho de los sedimentos con lo que se incrementaría su concentración, enriquecimiento y toxicidad (Coz et al. 2008, Karlsson et al. 2010).

Los sedimentos correspondientes a la presa Alzate, presentaron un $\mathrm{pH}$ promedio de 7.2. Respecto al COT se observa una disminución en la concentración 
en relación con el curso de agua, siendo a la entrada de $6.2 \%$ y a la salida de $0.8 \%$. Esto se debe a que en este embalse se degrada gran parte de la materia orgánica que transporta el río Lerma, así como a que el sedimento fino, al que está asociado la materia orgánica, es menor a $5 \%$ (Zarazúa 2008). Cabe mencionar que los sedimentos correspondientes a la salida (R12) presentan una concentración de $\mathrm{EC}_{50} 3.7$ veces mayor a los sedimentos de la entrada y centro (R10 y $\mathrm{R} 11)$, lo que sugiere una mejora en las condiciones de calidad de los sedimentos. Esto ya ha sido observado en estudios anteriores, en los que se concluyó que los sedimentos de esta presa actúan como un área de deposición de contaminantes y nutrientes contenidos en la columna de agua. Así, este embalse actúa como una laguna de estabilización en la que se están llevando a cabo procesos de sedimentación de contaminantes (Ávila et al. 1999, Ávila 2001, Carreño 2008, López et al. 2010, Barceló et al. 2012).

En cuanto a los sedimentos tomados en los tributarios al CARL, desde el canal Tenango hasta el arroyo Tototepec así como los del río Verdiguel, no muestran diferencias estadísticamente significativas entre ellos $(\alpha>0.05)$. Probablemente estos tributarios transportan aguas residuales de origen industrial con alto contenido de materia orgánica, lo cual se refleja en la concentración del COT que varió entre $6.4 \%$ y $10.6 \%$. Por otro lado, presentan bajos caudales, entre $0.04 \mathrm{~m}^{3} / \mathrm{s}$ y $0.36 \mathrm{~m}^{3} / \mathrm{s}$, acumulación de residuos sólidos y lirio acuático, lo que favorece la precipitación de compuestos orgánicos e inorgánicos que alteran la calidad de los sedimentos (Hinojosa 2006, Beg y Ali 2008, Ávila et al. 2011, Zarazúa et al. 2011). Sin embargo, los sedimentos de estos tributarios son estadísticamente diferentes a los de los ríos Xonacatlán, Santa Catarina, San Lorenzo, Tejalpa y Temoaya $(\alpha \leq 0.05)$, que transportan aguas residuales de origen doméstico y tienen caudales promedio entre $0.55 \mathrm{~m}^{3} / \mathrm{s}$ y $1.88 \mathrm{~m}^{3} / \mathrm{s}$, lo que contribuye a disminuir su toxicidad. Cabe mencionar que los sedimentos de estos ríos presentan una toxicidad semejante, es decir, no existen diferencias estadísticamente significativas entre ellos $(\alpha>0.05)$.

Respecto a la temporalidad, no se observaron diferencias significativas en la toxicidad de los sedimentos entre la época de estiaje y la de lluvia para los sedimentos del río ni para los de los tributarios $(\alpha>0.05)$. Este resultado concuerda con lo reportado por Tejeda et al. (2006) que indican que no hay diferencia significatitiva temporal en la concentración de metales en el sedimento depositado del CARL, lo cual se debe principalmente al método de muestreo aplicado que no permite la separación de estratos.
El CARL es un cuerpo de agua que debido a las descargas de aguas residuales urbanas e industriales no tratadas que recibe ha sobrepasado su capacidad de asimilación y dilución, por lo que se ha provocado su eutrofización, así como que el índice de calidad para la pesca y protección de la vida acuática sea inaceptable. Dadas las condiciones de bajo caudal y anoxia, es probable que los contaminantes se estén depositando, lo que ocasiona que los sedimentos del CARL se considereren como extremadamente tóxicos. Estas condiciones adversas han propiciado la alteración de las cadenas tróficas y la desaparición de peces, ajolotes y plantas, sobreviviendo solamente aquellos que son altamente resistentes como el lirio acuático (Eichhornia crassipes), lentejilla (Lemna sp.) algunas especies de nematodos, artrópodos, sanguijuelas, gasterópodos, plancton, entre otros (Ávila 2001, Tejeda et al. 2010, Zarazúa et al. 2013).

\section{CONCLUSIONES}

De acuerdo con la toxicidad obtenida por Microtox $\AA$, los sedimentos del CARL y sus principales tributarios se clasifican como extremadamente tóxicos. Los sedimentos correspondientes a los ríos Santa Catarina y Tejalpa se clasifican como moderadamente tóxicos y los de Temoaya y San Lorenzo se pueden considerar como no tóxicos.

No se observaron diferencias significativas en la toxicidad entre la época de estiaje y la de lluvia para los sedimentos en estudio, sin embargo sí se presentaron diferencias espaciales lo cual refleja la influencia de los aportes de origen principalmente antrópico en la parte media del CARL.

El análisis de la toxicidad de los sedimentos del CARL y sus tributarios refleja una situación ambiental grave y compleja ya que recibe la descarga de más de 52 afluentes de origen antropogénico (aguas industriales y municipales) y natural (arrastres pluviales y fluviales), por lo que este sistema hídrico requiere acciones de prevención, control y remediación de manera prioritaria.

\section{REFERENCIAS}

Ávila-Pérez P., Balcázar M., Zarazúa-Ortega G., BarcelóQuintal I. y Díaz-Delgado C. (1999). Heavy metal concentrations in water and bottom sediments of a Mexican reservoir. Sci. Total Environ. 234 (1-3), 185-196.

DOI: $10.1016 / \mathrm{S} 0048-9697(99) 00258-2$ 
Ávila P. (2001). Dinámica de metales pesados no esenciales en la interacción agua-sedimento-biota de la presa José Antonio Alzate. Tesis de Doctorado. Centro Interamericano de Recursos del Agua. Facultad de Ingeniería. Universidad Autónoma del Estado de México. Toluca, Estado de México, México, 197 pp.

Ávila-Pérez P., Zarazúa G., Carapia L., Tejeda S., BarcelóQuintal y Martínez T. (2011). Concentration of elements suspended matter discharges to Lerma River, Mexico. J. Radioanal. Nucl. Chem. 287 (2), 383-391. DOI: $10.1007 / \mathrm{s} 10967-010-0887-8$

AZUR (1992). Microtox manual. Microbics Corporation, AZUR Environmental, Carlsbad, California, EUA, $32 \mathrm{pp}$.

AZUR (1998). Basic solid-phase test (BSPT). AZUR Environmental, Carlsbad, California, EUA, 16 pp.

Barceló I., Solis R., Ávila P., López E., Gómez S. y García J. (2012). Determination of distributions of $\mathrm{Cd}, \mathrm{Cu}$ and $\mathrm{Pb}$ concentrations in sediments of a Mexican Reservoir to infer their environmental risk. Biol. Trace Elem. Res. 148 (1), 122-132. DOI: 10.1007/s12011-012-9458-x

Beelen P.V. (2003). A review on the application of microbial toxicity tests for deriving sediment quality guidelines. Chemosphere 53 (8), 795-808.

DOI: 10.1016/S0045-6535(03)00716-1

Beg K. y Ali S. (2008). Microtox toxicity assay for the sediment quality assessment of Ganga River. Am. J. Environ. Sci. 4 (4), 383-387.

Bennett J. y Cubbage J. (1992). Review and evaluation of Microtox test for freshwater sediments. Washington State Department of Ecology. Olympia Washington, EUA, $37 \mathrm{pp}$.

Bernet D., Schmidt P.H., Wahli T. y Burkhardt H.P. (2004). Evaluation of two monitoring approaches to assess effects of waste water disposal on histological alterations in fish. Hydrob. 524 (1), 53-66.

DOI: 10.1023/B:HYDR.0000036196.84682.27

Burga-Pérez K.F., Charlatchka R., Sahli L. y Férard J.F. (2012). New methodological improvements in the Microtox ${ }^{\circledR}$ solid phase assay. Chemosphere 86 (1), 105-110.

DOI: 10.1016/j.chemosphere.2011.08.042

Butterworth F., Guzmán J. y Corkum L. (1995). Biomonitors and biomarkers as indicators of environmental change: A Handbook. Plenum Press. Nueva York, EUA, 236 pp.

Bryan G. y Hummerstone L. (1978). Heavy metals in the burrowing bivalve Scrobicularia plana from contaminated and uncontaminated stuaries. J. Mar. Biol. Assoc. U.K. 58 (2), 401-419.

DOI: $10.1017 / \mathrm{S} 0025315400028071$

Bryan G. y Uysal H. (1978). Heavy metals in the burrowing bivalve Scrobicularia plana from the Tamara
Stuary in relation to environmental levels. J. Mar. Biol. Assoc. U.K. 58 (1), 89-108.

DOI: $10.1017 / \mathrm{S} 0025315400024437$

Campisi T., Abbondanzi F., Casado-Martínez C., DelValls T., Guerra R. y Iacondini A. (2005). Effect of sediment turbidity and color on light output measurement for Microtox ${ }^{\circledR}$ basic solid-phase test. Chemosphere 60 (1), 9-15.

DOI: 10.1016/j.chemosphere.2004.12.052

Casado-Martínez M.C., Campisi T., Díaz A., Lo Re R., Obispo R., Postma J.F., Riba I., Sneekes A.C., Buceta J.L. y DelValls T.A. (2006). Ejercicio interlaboratorio de bioensayos marinos para la evaluación de la calidad ambiental de sedimentos costeros en España. II. Ensayo de inhibición de la bioluminiscencia para la evaluación rápida de la toxicidad de sedimentos. Ciencias Marinas 32, 129-138.

Carreño C. (2008). Presencia y distribución de los compuestos orgánicos persistentes en agua y sedimento del curso alto del río Lerma. Tesis de Doctorado. Centro Interamericano de Recursos del Agua. Facultad de Ingeniería. Universidad Autónoma del Estado de México. Toluca, Estado de México, México, 234 pp.

Coz A., Rodríguez-Obeso O., Alonso-Santurde R., Álvarez-Guerra M., Andrés A., Viguri J.R., Mantzavinos D. y Kalogerakis N. (2008). Toxicity bioassays in core sediments from the Bay of Santander, Northern Spain. Environ. Res. 106 (3), 304-312.

DOI: 10.1016/j.envres.2007.05.009

Díaz C., Khalidou M., Iturbe A., Esteller M. y Reyna F. (2005). Características fisiográficas de una cuenca. En: Recursos hídricos: conceptos básicos y estudios de caso en Iberoamérica. (C. Díaz-Delgado, M. Esteller, F. López-Vera, Eds.). Piriguazú, Toluca, México, pp. 5-17.

Doe K., Jackman P., Scroggins R., McLeay D. y Wohlgeschaffen G. (2005). Solid-phase test for sediment toxicity using the luminescent bacterium, Vibrio fischeri. Small-scale freshwater toxicity investigations. En: Small-scale freshwater toxicity investigations toxicity test methods (C. Blaise, J. F. Férard, Eds.) Springer, Amsterdam, Países Bajos, pp. 107-136.

DOI: $10.1007 / 1-4020-3120-3$

GEM (1993). Atlas ecológico de la cuenca hidrográfica del río Lerma. Tomo I: Cartografía. Comisión Coordinadora para la Recuperación Ecológica de la Cuenca del Río Lerma. Gobierno del Estado de México. Toluca, México, 415 pp.

GEM (1997). Atlas ecológico de la cuenca hidrográfica del río Lerma. Tomo III: Ecosistemas Reproductivos. Comisión Coordinadora para la Recuperación Ecológica de la Cuenca del Río Lerma. Gobierno del Estado de México. Toluca, México, 250 pp. 
GEM (2000). Atlas ecológico de la cuenca hidrográfica del río Lerma. Tomo V: Industrial. Comisión Coordinadora para la Recuperación Ecológica de la Cuenca del Río Lerma. Gobierno del Estado de México. Toluca, México, 353 pp.

GEM (2002). Atlas ecológico de la cuenca hidrográfica del río Lerma. Tomo VII: Urbano. Comisión Coordinadora para la Recuperación Ecológica de la Cuenca del Río Lerma. Secretaría de Desarrollo Urbano y Obras Públicas, Gobierno del Estado de México. Toluca, México, 107 pp.

GEM (2009). Acciones realizadas para la recuperación ecológica de la cuenca del río Lerma 2008. Comisión Coordinadora para la Recuperación Ecológica de la Cuenca del Río Lerma. Gobierno del Estado de México. Toluca, México, $97 \mathrm{pp}$.

Fall C., Hinojosa P. y Carreño C. (2007). Design of a monitoring network and assessment of the pollution on the Lerma River and its tributaries by wastewaters disposal. Sci. Total Environ. 373 (1), 208-219.

DOI: 10.1016/j.scitotenv.2006.10.053

Hansen A., León A. y Bravo L. (1995). Fuentes de contaminación y enriquecimiento de metales en sedimentos de la cuenca Lerma-Chapala. Ing. Hidrául. Méx. 10 (3), 55-69.

Hattink J., De Boeck G. y Blust R. (2006). Toxicity, accumulation and retention of zinc by carp under normoxic and hipoxic conditions. Environ. Toxicol. Chem. 25 (1), 87-96. DOI: 10.1897/04-355R.1

Hinojosa A. (2006). Diseño de una red estratégica de monitoreo para el curso alto del río Lerma y su utilización en el estudios espacial y temporal de los parámetros físicoquímicos. Tesis de Maestría. Centro Interamericano de Recursos de Agua. Facultad de Ingeniería. Universidad Autónoma del Estado de México. Toluca, Estado de México, México, 186 pp.

Hsieh C.Y., Tsai M.H., Ryan D.K. y Pancorbo O.C. (2004). Toxicity of the 13 priority pollutant metals to Vibrio fisheri in the Microtox chronic toxicity test. Sci. Total Environ. 320 (1), 37-50.

DOI: 10.1016/S0048-9697(03)00451-0

Jennings V., Michael K., Rayner-Brandes H. y Bird D. (2001). Assessing chemical toxicity with the bioluminescent photobacterium (Vibrio fischeri): a comparison of three commercial systems. Water Res. 35 (14), 3448-3456.

DOI: 10.1016/S0043-1354(01)00067-7

Karlsson K., Viklander M., Scholes L. y Revitt M. (2010). Heavy metal concentrations and toxicity in water and sediment from stormwater ponds and sedimentation tanks. J. Hazard. Mater. 178 (1-3), 612-618.

DOI: 10.1016/j.jhazmat.2010.01.129

Kubitz J.A., Lewek E.C., Besser J.M., Drake J.B. y Giesy J.P. (1995). Effects of copper-contaminated sediments on Hyalella azteca, Daphnia magna and Cerodaphnia dubia: Survival, growth and enzime inhibition. Arch. Environ. Contam. Toxicol. 29 (1), 97-103.

DOI: 10.1007/BF00213093

Kwan K.K. y Dutka B.J. (1995). Comparative assessment of two solid-phase toxicity bioassays: The direct sediment toxicity testing procedure (DSTTP) and the Microtox ${ }^{\circledR}$ solid-phase test (SPT). Bull. Environ. Contam. Toxicol. 55 (3), 338-346. DOI: 10.1007/BF00206670

Kwan K.K. y Dutka B.J. (1996). Development of reference sediment samples for solid phase toxicity screening tests. Bull. Environ. Contam. Toxicology 56 (5), 696702. DOI: $10.1007 / \mathrm{s} 001289900102$

Levesque H., Dorval J., Hontela A., Van Der Kraak G. y Campbell P. (2003). Hormonal, morphological and physiological responses of yellow perch (Perca flavescens) to chronic environmental exposures. J. Toxicol. Environ. Health Part A 66 (7), 657-676.

DOI: $10.1080 / 15287390309353773$

López E., Barceló I., Solís C., Bussy A., Ávila P. y Martínez S. (2010). Calculation of the ecological risk index in the José Antonio Alzate Dam, State of Mexico, Mexico. Biol. Trace Elem. Res. 135 (1-3), 121-135. DOI: $10.1007 / \mathrm{s} 12011-009-8501-\mathrm{z}$

Luoma S. y Bryan G. (1979). Trace metal bioavailability: modeling chemical and biological interactions of sediment-bound Zinc. En: Chemical modeling in aqueous systems: speciation, sorption, solubility and kinetics. (R. F. Gould, Ed.). American Chemical Society, Washington, EUA, pp. 577-609.

DOI: 10.1021/bk-1979-0093.ch026

Mamindy-Pajanya Y., Hamerc B., Roméob M., Géretd F., Galganie F., Durmišif E., Hurela C. y Marmiera N. (2011). The toxicity of composted sediments from Mediterranean ports evaluated by several bioassays. Chemosphere 82 (3), 362-369.

DOI: 10.1016/j.chemosphere.2010.10.005

Mamindy-Pajany Y., Geret F., Roméo M., Hurel C. y Marmier N. (2012). Ex situ remediation of contaminated sediments using mineral additives: Assessment of pollutant bioavailability with the Microtox solid phase test. Chemosphere 86 (11), 1112-1116.

DOI: 10.1016/j.chemosphere.2011.12.001

Maranho L., Garrido-Pérez M., DelValls T. y Martín-Díaz M. (2015a). Suitability of standardized acute toxicity tests for marine sediment assessment: Pharmaceutical contamination. Water Air Soil Pollut. 226 (3), 1-14. DOI: $10.1007 / \mathrm{s} 11270-014-2273-6$

Maranho L., Garrido-Pérez M., Baena-Nogueras R., LaraMartín P., Antón-Martín R., DelValls T. y Martín-Díaz M. (2015b). Are WWTPs effluents responsible for acute toxicity? Seasonal variations of sediment quality at the Bay of Cádiz (SW, Spain). Ecotoxicol. 24 (2), 368-380. DOI: 10.1007/s10646-014-1385-5 
MEEO (1993). Guidelines for the protection and management of aquatic sediment quality in Ontario. Legislation policies, sediment classification and disposal options. Ministry of Environment and Energy of Ontario. Guideline. Toronto, Canada, 39 pp.

Morales-Caselles C., Kalman J., Riba I. y DelValls T. (2006). Comparing sediment quality in Spanish littoral areas affected by acute (Prestige, 2002) and chronic (Bay of Algeciras) oil spills. Environ. Pollut. 146 (1), 233-240.

DOI: $10.1016 /$ j.envpol.2006.04.042

Nyffeler U., Yuan L. y Santschi P. (1984). A kinetic approach to describe traceelement distribution between particles and solution in natural aquatic systems. Geochim. Cosmochim. Acta. 48 (7), 1513-1522.

DOI: 10.1016/0016-7037(84)90407-1

Onorati F. y Mecozzi M. (2004). Effects of two diluents in the Microtox ${ }^{\circledR}$ toxicity bioassay with marine sediments. Chemosphere 54 (5), 679-687.

DOI: 10.1016/j.chemosphere.2003.09.010

Pedroza S., Bussy A., Barceló I., López E. y Ávila P. (2010). Organic matter biodegradation and metal behavior in contaminated freshwater sediments. Int. J. Environ. Technol. Manage. 12 (1), 85-94. DOI: $10.1504 /$ IJETM.2010.029982

Riba I., Casado-Martínez M., Forja J. y DelValls T. (2004). Sediment quality in the Atlantic coast of Spain. Environ. Toxicol. Chem. 23 (2), 271-282.

DOI: $10.1897 / 03-146$

SCOFI (1995). Norma Mexicana. NMX-AA-112-1995SCFI: Análisis de agua y sedimentos - Evaluación de toxicidad aguda con Photobacterium phosphoreum Método de prueba. Secretaría de Comercio y Fomento Industrial. Diario Oficial de la Federación. 6 de marzo de 1996.

SMA (2006). Acuerdo por el que se da a conocer el estudio técnico de los recursos hídricos del área geográfica Lerma-Chapala. Secretaría de Medio Ambiente y Recursos Naturales. 24 de julio de 2006.

Suedel B., Deaver E. y Rodgers J. (1996). Experimental factors that may affect toxicity of aqueous and sediment-bound copper to freshwater organisms. Arch. Environ. Contam. Toxicol. 30 (1), 40-46.

DOI: $10.1007 / \mathrm{BF} 00211327$

Talapatra S.N. y Banerjee S.K. (2007). Detection of micronucleus and abnormal nucleus in erythrocytes form the gill and kidney of Labeo bata cultivated in sewagefed fish farms. Food Chem. Toxicol. 45 (2), 210-215. DOI: $10.1016 /$ j.fct.2006.07.022

Tarvainen T., Lahermo P. y Mannio J. (1997). Sources of trace metals in streams and headwater lakes in Finland. Water Air Soil Pollut. 94 (1-2), 1-32.

DOI: $10.1007 / \mathrm{BF} 02407090$
Tejeda S., Ávila-Pérez P., Zarazúa G., Carapia-Morales L., Díaz-Delgado C. y Lugo J. (2005). Distribución de metales pesados en sedimentos: estudio del curso alto del río Lerma. En: La medición de sedimentos en México. (F. Rivera-Trejo, A. Gutiérrez-López, R. Val-Segura, R. Mejía-Zermeño, P. Sánchez Ruíz, J. Aparicio-Mijarez, L. Díaz-Flores Eds.). Instituto Mexicano de Tecnología del Agua-Universidad Juárez Autónoma de Tabasco, Morelos/Tabasco, México, pp. 298-318.

Tejeda S., Zarazúa G., Avila-Pérez P., García-Mejía A., Carapia-Morales L. y Díaz-Delgado C. (2006). Major and trace elements in sediments of the upper course of Lerma River. J. Radioanal. Nucl. Chem. 270 (1), 9-14. DOI: 10.1007/s10967-006-0342-z

Tejeda S., Zarazúa G., Ávila-Pérez P., Carapia-Morales L. y Martínez T. (2010). Total reflection x-ray fluorescence spectrometric determination of elements in water hyacinth from the Lerma River. Spectroch. Acta B. 65 (6), 483-488.

DOI: $10.1016 /$ j.sab.2010.04.005

Tsiridis V., Petala M., Samaras P., Hadjispyrou S., Sakellaropoulos G. y Kungolos A. (2006). Interactive toxic effects of heavy metals and humic acids on Vibrio fischeri. Ecotox. Environ. Safe. 63 (1), 158-167. DOI: 10.1016/j.ecoenv.2005.04.005

Walton V., Compton S., Allan J. y Daniels R. (1982). The effect of acid stress on survivonship and reproduction of Daphnia pulex (Crustacea: Cladocera). Can. J. Zoo. 60 (4), 573-579. DOI: 10.1139/z82-085

Zarazúa G., Ávila-Pérez P., Tejeda S., Barceló-Quintal I. y Martínez T. (2006). Analysis of total and dissolved heavy metals in surface water of a Mexican polluted river by TXRF. Spectroch. Acta B. 61 (10-11), 11801184. DOI: $10.1016 /$ j.sab.2006.06.010

Zarazúa G. (2008). Evaluación de las contribuciones naturales y antropogénicas de los metales pesados $\mathrm{Cr}, \mathrm{Mn}, \mathrm{Fe}, \mathrm{Cu}, \mathrm{Zn}$ y $\mathrm{Pb}$ y su distribución en el agua y sedimento en el curso alto del río Lerma. Tesis de Doctorado. Centro Interamericano de Recursos de Agua. Facultad de Ingeniería. Universidad Autónoma del Estado de México. Toluca, Estado de México, México, $255 \mathrm{pp}$.

Zarazúa G., Tejeda S., Ávila P., Carapia L., Carreño C. y Balcázar M. (2011). Metal content and elemental composition of particles in cohesive sediments of the Lerma River, Mexico. Rev. Int. Contam. Ambie. 27 (3), 181-190

Zarazúa G., Ávila-Pérez P., Tejeda S., Valdivia-Barrientos M., Zepeda-Gómez C. y Macedo-Miranda G. (2013). Evaluación de los metales pesados $\mathrm{Cr}, \mathrm{Mn}, \mathrm{Fe}, \mathrm{Cu}, \mathrm{Zn}$ y $\mathrm{Pb}$ en sombrerillo de agua (Hydrocotyle ranunculoides) del curso alto del río Lerma, México. Rev. Int. Contam. Ambie. 29 (Sup. 2), 217-24 